\title{
Development and evaluation of next-generation cardiotoxicity assay based on embryonic stem cell-derived cardiomyocytes
}

\author{
Bokyeong Ryu ${ }^{1}$, Seong Woo Choi ${ }^{2}$, Seul-Gi Lee ${ }^{2}$, Young-Hoon Jeong ${ }^{2}$, Ukjin Kim ${ }^{1}$, Jin Kim ${ }^{1}$, Cho-Rok Jung ${ }^{3}$, \\ Hyung-Min Chung ${ }^{2}$, Jae-Hak Park ${ }^{1}$ \& C-Yoon Kim ${ }^{2, *}$ \\ ${ }^{1}$ Department of Laboratory Animal Medicine, College of Veterinary Medicine, Seoul National University, Seoul 08826, ${ }^{2}$ Department of \\ Medicine, School of Medicine, Konkuk University, Seoul 05029, ${ }^{3}$ Gene Therapy Research Unit, Korea Research Institute of Bioscience and \\ Biotechnology, Daejeon 34141, Korea
}

\begin{abstract}
In accordance with requirements of the ICH S7B safety pharmacology guidelines, numerous next-generation cardiotoxicity studies using human stem cell-derived cardiomyocytes (CMs) are being conducted globally. Although several stem cell-derived CMs are being developed for commercialization, there is insufficient research to verify if these $\mathrm{CMs}$ can replace animal experiments. In this study, in vitro high-efficiency $\mathrm{CMs}$ derived from human embryonic stem cells (hESC-CMs) were compared with SpragueDawley rats as in vivo experimental animals, and primary cultured in vitro rat-CMs for cardiotoxicity tests. In vivo rats were administrated with two consecutive injections of $100 \mathrm{mg} /$ $\mathrm{kg}$ isoproterenol, $15 \mathrm{mg} / \mathrm{kg}$ doxorubicin, or $100 \mathrm{mg} / \mathrm{kg}$ nifedipine, while in vitro rat-CMs and hESC-CMs were treated with $5 \mu \mathrm{M}$ isoproterenol, $5 \mu \mathrm{M}$ doxorubicin, and $50 \mu \mathrm{M}$ nifedipine. We have verified the equivalence of hESC-CMs assessments over various molecular biological markers, morphological analysis. Also, we have identified the advantages of hESC-CMs, which can distinguish between species variability, over electrophysiological analysis of ion channels against cardiac damage. Our findings demonstrate the possibility and advantage of high-efficiency hESC-CMs as next-generation cardiotoxicity assessment. [BMB Reports 2020; 53(8): 437-441]
\end{abstract}

\section{INTRODUCTION}

Cardiac toxicity is a major cause of failure in drug development (1). Also, drug-induced arrhythmia is the most common cause of restrictions or withdrawal of new drugs in the market. For these reasons, risk assessment for cardiovascular adverse

*Corresponding author. Tel: +82-2-453-9012; Fax: +82-2-455-9012; E-mail: vivavets@gmail.com

https://doi.org/10.5483/BMBRep.2020.53.8.022

Received 3 February 2020, Revised 28 February 2020, Accepted 28 February 2020

Keywords: Alternative, Cardiomyocyte, Drug withdrawal, Embryonic stem cell, Toxicity test effects is a crucial safety pharmacological element in new drug development. In the Regulatory Guides (2, 3), hERG (hKv11 potassium channel) assays and in vivo ECG assays are recommended to predict risk of arrhythmia associated with ventricular repolarization (QT prolongation) in humans.

Automated patch clamping is the standard method for high efficiency cardiac safety screening, since most clinical cases of drug-induced cardiac toxicity are associated with hERG inhibition. However, this approach has limitations because securing sufficient primary human myocardial cells and long-term cultivation are difficult. Additionally, QT intervals are the result of interdependent ion channel activity, and inhibitory potential in the hERG does not always lead to arrhythmia for the heart (4). To address this problem, Purkinje fibers of guinea pig or rabbit heartshave been used for patch clamping at later stages of the drug screening process to study the action potential (AP) and ECG changes. However, interspecies variation can interfere with reliable connections to clinical relevance.

As an alternative to primaryhuman myocardial cells and animal experiments, human stem cell-derived cardiomyocyte model systems have been developed as physiologically relevant models for cardiac toxicity testing (5-9). Because of their ability to form synchronously beating cell populations, stem cell-derived cardiomyocytes are very attractive in vitro models that can be used to reliably reproduce drug-related cardiovascular phenotypes (10). Of particular significance is the ability to use stem cellderived cardiomyocytes to screen for potential toxicities of chemicals that can cause cardiac arrhythmias as they express human ion channels similar to primary human cardiomyocytes and electrophysiological features similar to in vivo electrocardiography (11). Even in Comprehensive in vitro Proarrhythmia Assay (CiPA), developed as a next-generation cardiotoxicity model, cellular electrophysiological functions have been importantly evaluated upon drug effects (12). Although many laboratories, pharmaceutical companies, and research institutes have initiated human stem cell-derived cardiomyocytes research for cardiac toxicity assessments including CiPA (12-15), there is no preclinical study to evaluate if the patch clamping analysis of hERG channels using human embryonic stem cellderived cardiomyocytes (hESC-CMs) predicts drug-toxic effects

ISSN: 1976-670X (electronic edition)

Copyright (c) 2020 by the The Korean Society for Biochemistry and Molecular Biology

(c) This is an open-access article distributed under the terms of the Creative Commons Attribution Non-Commercial License (http://creativecommons.org/licenses/by-nc/4.0) which permits unrestricted non-commercial use, distribution, and reproduction in any medium, provided the original work is properly cited. 
compared with standardization techniques such as in vivo animal ECG and hERG assays, which use non-cardiac cell lines overexpressed with the human ion channels (heterologous expression systems) or the disaggregated cardiomyocytes.

In this study, we compared and reported drug-induced cardiac toxicity to characteristic drugs using our high purity hESC-CMs reported in a previous study. For this purpose, we verified various gene panel expression changes and electrophysiological changes of hESC-CMs against animal cell and tissue data. Our study demonstrates that the hESC-CMs are an effective alternative testing method, which can replace the animal cell-based hERG assay or in vivo animal ECG model.

\section{RESULTS}

\section{Characterization of hESC-CMs}

In this study, hESC-CMs were generated using a small moleculesbased differentiation method as previously reported (16-18) (Fig. 1A). Spontaneous beating was observed around differentiation days 10-15. Immunostaining for hESC-CMs was performed on day 30 by staining for cardiac specific markers, cardiac troponin $\mathrm{T}(\mathrm{cTnT})$ and sarcomeric $\alpha$-actinin-2 (Fig. 1B) and approximately $80 \%$ of purity was confirmed by flow cytometry (Fig. 1C). On day 30, besides, the beating was recorded in video (Supplementary Video 1) and in microelectrode array (MEA) (Supplementary Fig. 1). Developed hESC-CMs were verified to meet the CiPA criteria (12).

\section{Morphological changes in three models}

With isoproterenol, doxorubicin, and nifedipine, morphological changes were observed (Fig. 2A). In isoproterenol and doxoru-
(A)

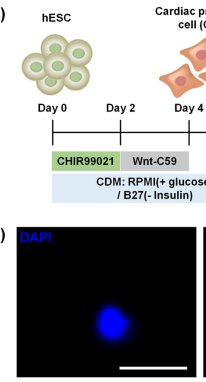

(C)

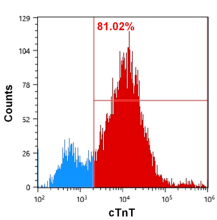

Fig. 1. Overview of the hESC-CM differentiation. (A) The hESC-CM differentiation protocol using small-molecules. (B) At differentiation day 30, the cells were stained with cTnT and $\alpha$-actinin-2. Scale bars: $100 \mu \mathrm{m}$. (C) And at day 30, the purity was monitored by the flow cytometry and showed as upper $80 \%$. bicin, rat hearts exhibited damaged cardiac myocyte architectures with cell shrinkage, nuclear pyknosis, karyolysis, densed cytoplasm, and vacuolation. Coinciding with the histological damages, the cells with isoproterenol and doxorubicin became irregular and exhibited shrinkage and detachment of the cells from cell culture substratum, indicating apoptosis. Additionally, deterioration of $\alpha$-actinin-2 arrangement was observed in all three models with isoproterenol or doxorubicin (Fig. 2B). Apoptotic indices were also calculated in rat hearts (Fig. 2C), rat-CMs (Fig. 2D), and the hESC-CMs (Fig. 2E) using the TUNEL assay (Supplementary Fig. 2). In the TUNEL results, isoproterenol and doxorubicin showed significant apoptotic cell increases in all models.

Relative changes of mRNA expression related to cytotoxicity To assess the general cytotoxic response to drug-induced toxicity, the transcript levels of oxidative stress, cardiotoxicity, inflammation, and apoptosis-related genes were monitored (Fig. 3A). The oxidative stress indicators, iNOS, Hmox1, Sod1, and Gpx1 levels increased in all models with all drugs. Additionally, the cardiotoxicity-related gene levels, $\beta-\mathrm{MHC}$, ANP,

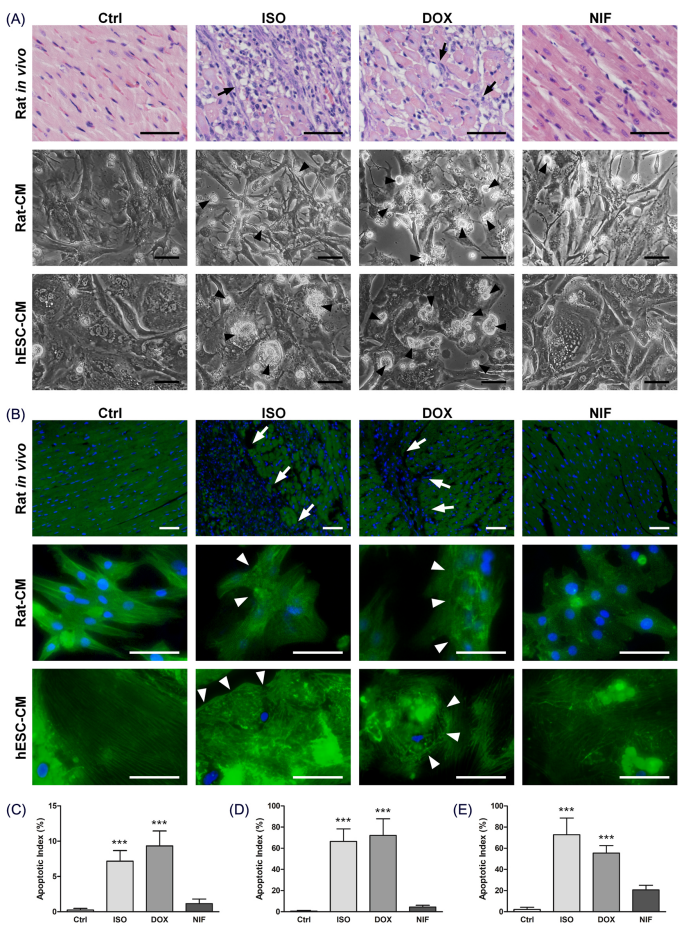

Fig. 2. Morphological changes in three models with cardiotoxicants. (A) The rat hearts showed damaged cardiac myocyte architectures (arrows), and characteristics of apoptotic cell death showed in the cells (arrowheads). Scale bars: $50 \mu \mathrm{m}$. (B) All of samples were stained with $\alpha$-actinin-2 (green) and DAPI (blue). The arrows and arrowheads indicate $\alpha$-actinin-2 deterioration. Scale bars: $100 \mu \mathrm{m}$. (C-E) The apoptosis indices of rats (C), rat-CMs (D), and hESC-CMs (E) were assessed with the TUNEL. Data shown are mean \pm SD $(* * * P<0.001$; $\mathrm{n}=5$ ). 


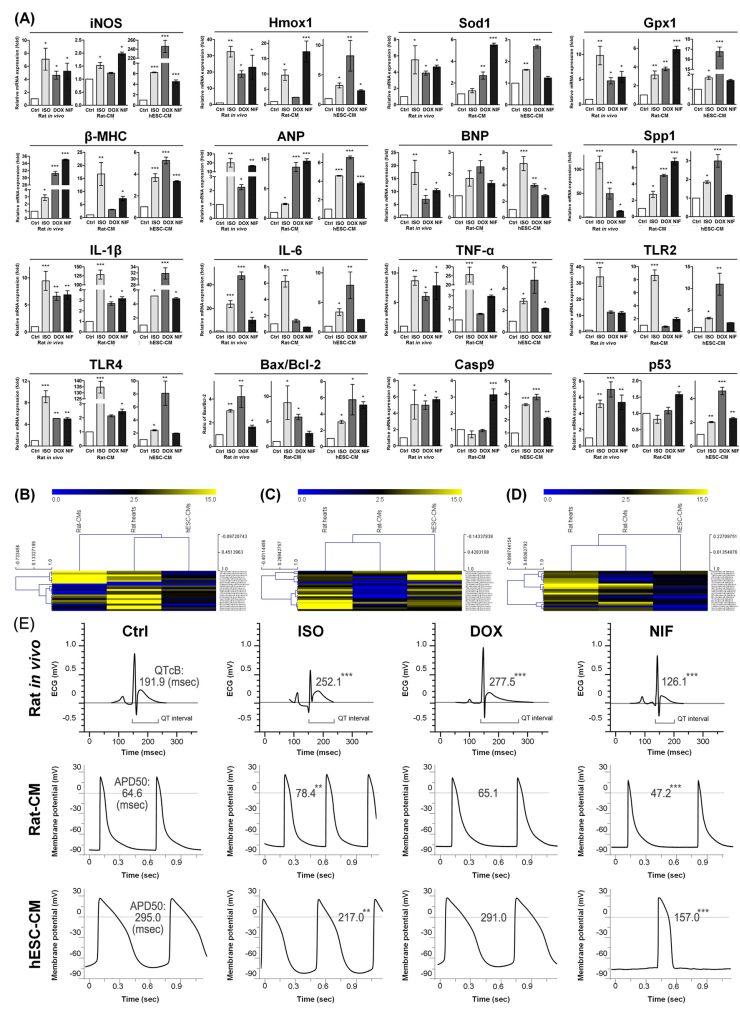

Fig. 3. mRNA expression and electrophysiology in three models with cardiotoxicants. (A) The relative expression of iNOS, Hmox1, Sod1, Gpx1, $\beta-M H C$, ANP, BNP, Spp1, IL-1 $\beta$, IL-6, TLR2, TLR4, TNF- $\alpha$, ratio of $\mathrm{Bax} / \mathrm{BCl}-2$, Casp9, p53 were monitored. Data shown are mean \pm SEM (*, $\mathrm{P}<0.05 ; * *, \mathrm{P}<0.01 ; * * *, \mathrm{P}<0.001 ; \mathrm{n}=5)$. (B-D) The heat-maps in which the entire set of genes were clustered hierarchically to visualize general expression patterns with isoproterenol (B), doxorubicin (C), or nifedipine (D). The profiles are represented by models (columns) and genes (rows). The color scale at the top represents relative expression level, wherein yellow and blue colors indicate upregulated and unaltered expression, respectively (See Supplementary Table 1). (E) The ECG and patch clamp were recorded (see Table 1).

BNP, and Spp1, and inflammation-related genes, IL-1 $\beta$, IL-6, TLR2, TLR4, and TNF- $\alpha$, were also increased in all groups. Also, indicators of apoptosis, the ratio of Bax/Bcl-2, and Casp9 and p53, increased significantly in all the groups.

Generally, nifedipine showed weak responses compared to the other drugs in all models. These results show the general cytotoxic response of hESC-CMs to cardiotoxicants is equivalent to rats and rat-CMs. Additionally, overall data was visualized as heat-maps to show general expression patterns of the three models by drugs (Fig. 3B-3D). Additionally, hESC-CMs showed relatively strong correlation with in vivo model with isoproterenol (Fig. 3B), while rats and rat-CMs showed similarity with the doxorubicin (Fig. 3C) or the nifedipine (Fig. 3D). The gene order is presented in Supplementary Table 1.

\section{Changes in electrophysiological profile}

For comparison of quantitative analysis of the cardiac function, beat rate, corrected QT (QTc) intervals, and AP durations (APD) during drug treatment were evaluated respectively. The QTC and APD represent electrical depolarization and repolarization of the ventricles, prolongation of which is a marker of a potential risk factor for sudden death. The QTc was calculated using Bazett's formula (QTcB), Fridericia's formula (QTcF), and Sagie's formula (QTIc) in all groups $(19,20)$. As shown in Fig. $3 \mathrm{E}$ and Table 1, rats and rat-CMs showed significant increases in the beat rate and prolongation of repolarization time with isoproterenol, a $\beta$-adrenoceptor agonist. hESC-CMs also showed a significant increase in the beat rates; however, APD of hESC-CMs showed the opposite response to the rat hearts and the rat-CMs. Importantly, this different response is associated with the expression of species-specific $\beta$-adrenoceptor subtypes. Next, there was no change in the beat rate with doxorubicin, an anticancer drug, which causes QTc prolongation, but theduration of repolarization was prolonged in the rats. Finally, when treated with the L-type calcium blocker, nifedipine, which causes bradycardia and hypotension, significant reductions in the beat rate and repolarization times were observed in all groups however, no change in amplitude was observed in any group. These results indicate that hESC-CMs are equally applicable as laboratory animal rats and rat cardiomyocytes for conventional cardiac pharmacokinetic studies. Also, given the species different response, it shows the possibility of an optimal tool that can be more suitable for humans.

\section{DISCUSSION}

Globally, a variety of drug screening platforms are being studied, and human stem cells have been evaluated as the best alternative to replace conventional animal testing. In particular, the ability to detect toxicity and efficacy of human species-specific responses, which can be missed in an animal model, is a major advantage of stem cells. We have reported a technique for high-efficient differentiation of hESC-CMs (21). In this study, we evaluated the technology of next-generation cardiotoxicity using hESC-CMs.

In this study, hESC-CMs showed very homogenous characteristics over a long period of culture, with the expression of cardiac-specific markers, likewise in the previous studies. The cultured hESC-CMs in sheet-shape additionally exhibited synchronized, uniform myocardial contraction, and stable characteristics in the electrophysiological analysis.

The rats, rat-CMs, and hESC-CMs were treated with reference drugs, isoproterenol, doxorubicin and nifedipine, which cause cardiac damage, respectively. For these cardiotoxicants, all three models showed generally similar responses in morphological and mRNA changes. While isoproterenol and doxorubicin caused significant damages to the tissues and cells, nifedipine showed weak responses, suggesting that nifedipine as a commercialized drug could be safe at concentrations below 
Table 1. Electrophysiological changes in three models with cardiotoxicants

\begin{tabular}{lcccc}
\hline & Ctrl & ISO & DOX & NIF \\
\hline ECG results from rats & & & & $224 \pm 22^{* * *}$ \\
HR (bpm) & $287 \pm 2$ & $452 \pm 17^{* * *}$ & $271 \pm 16$ & $65.31 \pm 7.78^{* *}$ \\
QT (msec) & $87.76 \pm 12.92$ & $91.84 \pm 0.52$ & $130.61 \pm 10.43^{* * *}$ & $126.06 \pm 15.01^{* * *}$ \\
QTcB (msec) & $191.87 \pm 28.24$ & $252.11 \pm 1.41^{* * *}$ & $277.52 \pm 22.16^{* * *}$ & $101.25 \pm 12.05^{* * *}$ \\
QTcF (msec) & $147.83 \pm 21.76$ & $180.06 \pm 1.01^{*}$ & $215.87 \pm 17.24^{* * *}$ & $177.98 \pm 7.78^{* * *}$ \\
QRlc (msec) & $209.54 \pm 12.92$ & $225.40 \pm 0.51^{*}$ & $250.50 \pm 10.43^{* * *}$ & $38 \pm 10^{* * *}$ \\
Patch clamp results from rat-CMs & $117 \pm 9$ & $142 \pm 13^{* *}$ & $114 \pm 10$ & $47.2 \pm 4.5^{* * *}$ \\
HR (bpm) & $64.6 \pm 5.2$ & $78.4 \pm 6.4^{* *}$ & $65.1 \pm 4.9$ & $64 \pm 8^{*}$ \\
APD50 (msec) & & & $157.0 \pm 38.0^{* * *}$ \\
Patch clamp results from hESC-CMs & & & $87 \pm 7$ & $291.0 \pm 21.4$ \\
HR (bpm) & $83 \pm 12$ & $217.0 \pm 22.1^{* *}$ & & \\
APD50 (msec) & $295.0 \pm 30.9$ &
\end{tabular}

Data shown are mean $\pm \mathrm{SD}(* \mathrm{P}<0.05 ; * * \mathrm{P}<0.01 ; * * * \mathrm{P}<0.001 ; \mathrm{n}=5)$. See Fig. $3 \mathrm{E}$.

the lethal dose 50. These results demonstrate hESC-CMs can assess cardiotoxicity at comparable levels to conventional animal experiments or primary cultured cells. To prove further correlations between hESC-CMs with conventional models, heat-maps were assessed. Particularly, hESC-CMs showed strong correlation with in vivo model in isoproterenol than doxorubicin or nifedipine. Correlations between hESC-CMs and in vivo model with doxorubicin or nifedipine, however, seemed weaker than correlations between the rat-CMs and rats.

Different responses between the models were observed, which could be attributed to metabolism and cell composition differences in species. Susceptibilities for oxidative stress, particularly, were somewhat different between the samples originating from other species. For Sod1 and Gpx1 with nifedipine, rats and rat-CMs showed significant increases unlike hESC-CMs. Conversely, for iNOS with doxorubicin, hESC-CMs showed more dramatic change rather than the rat samples. These phenomena could be elaborated with facts that Sod 1 and Gpx1 are directly impacted by ROS while iNOS is indirectly influenced via NF-KB. It could explain the different responding quickness for oxidative stress (22). Also, oxidative stress induces lipid peroxidation (23), producing products including malondialdehyde (24). Rats differ from humans within the membrane susceptibilities for these products $(25,26)$. Some differences between the rat samples and the hESC-CMs could be explained with facts about work-timing differences of enzymes in oxidative stress pathways and species differences of susceptibilities for oxidative stress products. Additionally, we observed critical differences by drugs in the electrophysiological results.

To verify the electrophysiological function of the hESC-CMs, we compared the three models. In the heart rate, increases by isoproterenol and decreases by nifedipine were significantly observed in all the models. Unlike morphological and mRNA analyses, doxorubicin did not impact electrophysiologically. Additionally, we analyzed cQT and APD, the main arrhythmia indicators, which correspond to myocardial depolarization and repolarization. CQT and APD reduction by nifedipine was observed in all the models. Importantly, hESC-CM APD was reduced with isoproterenol, unlike rat CQT and APD increase, suggested to be from species differences in $\beta$-adrenoceptor distribution. Ruzsnavszky, et al. (27) reported, in large mammals, $\beta 1$-adrenoceptors are distributed more dominantly than $\beta 2$-adrenoceptors and cAMP-dependent activities of $\mathrm{Ca}^{2+}$ and $\mathrm{K}^{+}$currents are different from those of small mammals. These results suggest that the hESC-CMs are capable of assessing cardiotoxicity closer to humans compared to conventional models.

Although further assessment of human-specific toxicants should be accompanied, based on our developed the hESC$\mathrm{CMs}$, our team will continue to develop the next-generation cardiotoxicity assay through continuous verification.

\section{CONCLUSION}

The developed hESC-CMs were verified to have superiority than the conventional models in the toxicological reactions molecularly, morphologically, and electrophysiologically, while the hESC-CM can distinguish between species variabilities. These results demonstrate the possibility and advantage of the next-generation cardiotoxicity assessment using high-efficiency hESC-CMs.

\section{MATERIALS AND METHODS}

See supplementary information for Material and Methods.

\section{ACKNOWLEDGEMENTS}

This study was supported by a grant from the KRIBB Research Initiative Program; the National Research Foundation of Korea [Grant/Award Number: NRF-2015M3A9C7030091 and NRF-2018 
R1C1B6007354]; a grant from the Research Institute of Veterinary Science, the College of Veterinary Medicine, Seoul National University; and a grant from the BK21 PLUS Program for Creative Veterinary Science Research, College of Veterinary Medicine, Seoul National University.

\section{CONFLICTS OF INTEREST}

The authors have no conflicting interests.

\section{REFERENCES}

1. Scannell JW, Blanckley A, Boldon $\mathrm{H}$ and Warrington $\mathrm{B}$ (2012) Diagnosing the decline in pharmaceutical R\&D efficiency. Nat Rev Drug Discov 11, 191-200

2. International Council of Harmonization (ICH) Guidelines (2005) The non-clinical evaluation of the potential for delayed ventricular repolarization (Qt Interval Prolongation) by human pharmaceuticals. S7B (https://database.ich. org/sites/default/files/S7B Guideline.pdf)

3. International Council of Harmonization $(\mathrm{ICH})$ Guidelines (2006) The clinical evaluation of QT/QTc interval prolongation and proarrhythmic potential for non-antiarrhythmic drugs. E14 4 (https://database.ich.org/sites/default/ files/E14 Guideline.pdf)

4. Hoffmann P and Warner B (2006) Are hERG channel inhibition and QT interval prolongation all there is in drug-induced torsadogenesis? A review of emerging trends. J Pharmacol Toxicol Methods 53, 87-105

5. Guo L, Coyle L, Abrams RM, Kemper R, Chiao ET and Kolaja KL (2013) Refining the human iPSC-cardiomyocyte arrhythmic risk assessment model. Toxicol Sci 136, 581-594

6. Mercola M, Colas A and Willems E (2013) Induced pluripotent stem cells in cardiovascular drug discovery. Circ Res 112, 534-548

7. Suter-Dick L, Alves PM, Blaauboer BJ et al (2015) Stem cell-derived systems in toxicology assessment. Stem Cells Dev 24, 1284-1296

8. Palpant NJ, Pabon L, Friedman CE et al (2017) Generating high-purity cardiac and endothelial derivatives from patterned mesoderm using human pluripotent stem cells. Nat Protoc $12,15-31$

9. Ronaldson-Bouchard K, Ma SP, Yeager K et al (2018) Advanced maturation of human cardiac tissue grown from pluripotent stem cells. Nature 556, 239-243

10. Sirenko O, Cromwell EF, Crittenden C, Wignall JA, Wright FA and Rusyn I (2013) Assessment of beating parameters in human induced pluripotent stem cells enables quantiative in vitro screening for cardiotoxicity. Toxicol Appl Pharmacol 273, 500-507

11. Blinova K, Dang Q, Millard D et al (2018) International Multisite Study of Human-Induced Pluripotent Stem CellDerived Cardiomyocytes for Drug Proarrhythmic Potential Assessment. Cell Rep 24, 3582-3592

12. Millard D, Dang Q, Shi H et al (2018) Cross-Site Reliability of Human Induced Pluripotent stem cell-derived Cardiomyocyte Based Safety Assays Using Microelectrode
Arrays: Results from a Blinded CiPA Pilot Study. Toxicol Sci 164, 550-562

13. Takasuna K, Asakura K, Araki S et al (2017) Comprehensive in vitro cardiac safety assessment using human stem cell technology: Overview of CSAHi HEART initiative. J Pharmacol Toxicol Methods 83, 42-54

14. Magdy T, Schuldt AJT, Wu JC, Bernstein D and Burridge PW (2018) Human Induced Pluripotent Stem Cell (hiPSC)Derived Cells to Assess Drug Cardiotoxicity: Opportunities and Problems. Annu Rev Pharmacol Toxicol 58, 83-103

15. Burridge PW, Li YF, Matsa E et al (2016) Human induced pluripotent stem cell-derived cardiomyocytes recapitulate the predilection of breast cancer patients to doxorubicininduced cardiotoxicity. Nat Med 22, 547-556

16. Burridge PW, Matsa E, Shukla P et al (2014) Chemically defined generation of human cardiomyocytes. Nat Methods $11,855-860$

17. Lian X, Hsiao C, Wilson G et al (2012) Robust cardiomyocyte differentiation from human pluripotent stem cells via temporal modulation of canonical Wnt signaling. Proc Natl Acad Sci U S A 109, E1848-1857

18. Minami I, Yamada K, Otsuji TG et al (2012) A small molecule that promotes cardiac differentiation of human pluripotent stem cells under defined, cytokine- and xenofree conditions. Cell Rep 2, 1448-1460

19. Vandenberk B, Vandael E, Robyns T et al (2016) Which QT Correction Formulae to Use for QT Monitoring? J Am Heart Assoc 5, e003264

20. Sagie A, Larson MG, Goldberg RJ, Bengtson JR and Levy D (1992) An improved method for adjusting the QT interval for heart rate (the Framingham Heart Study). Am J Cardiol 70, 797-801

21. Park SJ, Bae D, Moon SH and Chung HM (2013) Modification of a purification and expansion method for human embryonic stem cell-derived cardiomyocytes. Cardiology 124, 139-150

22. Lee JY, Kim MY, Shin SH et al (2017) Persicarin isolated from Oenanthe javanica protects against diabetes-induced oxidative stress and inflammation in the liver of streptozotocin-induced type 1 diabetic mice. Exp Ther Med 13, 1194-1202

23. Songbo $M$, Lang $H$, Xinyong $C$, Bin $X$, Ping $Z$ and Liang $S$ (2019) Oxidative stress injury in doxorubicin-induced cardiotoxicity. Toxicol Lett 307, 41-48

24. Ayala A, Munoz MF and Arguelles S (2014) Lipid peroxidation: production, metabolism, and signaling mechanisms of malondialdehyde and 4-hydroxy-2-nonenal. Oxid Med Cell Longev 2014, 360438

25. Janssen M, Koster JF, Bos E and de Jong JW (1993) Malondialdehyde and glutathione production in isolated perfused human and rat hearts. Circ Res 73, 681-688

26. Singh Y, Hall GL and Miller MG (1992) Species differences in membrane susceptibility to lipid peroxidation. J Biochem Toxicol 7, 97-105

27. Ruzsnavszky F, Hegyi B, Kistamas K et al (2014) Asynchronous activation of calcium and potassium currents by isoproterenol in canine ventricular myocytes. Naunyn Schmiedebergs Arch Pharmacol 387, 457-467 